\title{
State of Charge Estimation in Lithium-Sulfur Cells using LSTM Recurrent Neural Networks*
}

\author{
Zhihuo Wang, Abbas Fotouhi and Daniel J. Auger
}

\begin{abstract}
This paper presents a framework for all-state estimation of Lithium-Sulfur ( $\mathrm{Li}-\mathrm{S})$ battery cells based on a Long Short-Term Memory Recurrent Neural Network (LSTM RNN) model. Under the proposed framework, the LSTM RNN model is calibrated into the single task of State of Charge (SoC) estimation for fresh Li-S prototype cells. The Adaptive Moment Estimation (Adam) solver is used. Data sets for training and testing are derived from experiments using the WLTP duty cycles. The calibrated LSTM RNN structure is described for the purposes of training and testing with experimental datasets, so as to generate a network that can be deployed in real-time system. The demonstration of the training and testing results has shown robustness of the proposed approach against nonlinearities of the experimental datasets and uncertainty in initial SoC. The approach gave satisfactory estimation performance with an acceptable tradeoff between estimation accuracy and convergence speed.
\end{abstract}

\section{INTRODUCTION}

As the battery industry develops, research challenges are arising in relation to the next generation of battery cells. This encompasses many areas such as electrode materials, manufacturing, lifetime extension, safety enhancement technologies, multi-scale modelling, battery management systems and BMS. In the UK alone, the Faraday Battery Challenge has funded approximately 100 business entities and 20 research organizations working to fulfill these technical gaps and deliver world-class innovations in automotive battery technology [1]. There are no doubt similar efforts worldwide.

Energy density and safety are key concerns of battery industry. Lithium sulfur (Li-S) cells offer higher gravimetric energy density and volumetric energy density compared with commercial lithium ion. Commercial organizations have patented materials designed for safety, allowing $\mathrm{Li}-\mathrm{S}$ cells to meet international standards criteria in terms of abuse testing.: existing Li-S cells can withstand extreme abuse situations within commercial application scenarios, which offers potential for a high safety level in commercial use [2]. Li-S can be regarded as a promising technology with distinct advantages over alternatives in the future market. Current research aims to eliminate the remaining limitations such as poor instantaneous power capability and short cycle life [3].

*Research supported by the European Commission (ALISE, Horizon 2020 grant agreement no. 666157) and Innovate UK (LiS:FAB, TS/R013780/1). The data used in this article is described in CORD at https://doi.org/10.17862/cranfield.rd.11843430; it is subject to an embargo, and will be available from 1 April 2031.

The authors are with Cranfield University, Cranfield, MK43 OAL, UK (email: d.j.auger@cranfield.ac.uk).
To gain full advantage of Li-S, it it is vital to understand its in-application behavior. This has imposed specific requirements on modelling and state observation issues. Multiplescale modelling of the Li-S cells have been carried out from atoms to structure, to cell, module and pack design. A series of Li-S cell state monitoring and control methods depending on the battery cell model has been developed, including a set of modeling and parameterization methods considering scenarios such as charging, discharging, thermal effect, and aging effects. [4, 5, 6, 7]. These methods have been verified through tests and experiments, and finally applied in a BMS. Accurate battery models capable of predicting all dynamic characteristics is important, and a key research gap has been the predictability of all the relevant parameters to quantify performance in the presence of ageing.

The most representative unknown states in batteries are the SoC, remaining useful life, state of power and state of health, which has attracted a lot of researchers in recent years $[8,9]$. These indicators depend on the internal performance of the battery, and they are have non-linear relations to electrical, chemical and thermal parameters. Equivalent circuit models with unknown parameters have been built to solve the state estimation problems, however, these relies on the parameter identification algorithms which can be vulnerable to inaccuracies and add to the online calculation burden. More overall, the involvement of several polysulfide species and its solubility during discharge led to a relatively stable voltage plateaulow plateau, which causes challenges in the observability of SoC. This is a distinctive feature of Li-S cell which distinguishes it from many other types of battery cell $[10,11]$. The requirement for a relatively accurate initial $\mathrm{SoC}$ value can be a limitation for some traditional SoC estimation methods.

Artificial Neural Networks (ANNs) have provided an alternative approach which does not require much prior knowledge of the Li-S cell. ANNs have been used on a variety of tasks including computer vision, speech recognition, machine translation, medical diagnosis, and so on. The basic ANN algorithm proposes a candidate model which can be evaluated against experimental datasets and an ANN structure in accordance with working scenarios. By choosing candidate networks and algorithms, an ANN is capable of carrying out regression analysis, sequence recognition, clustering, and numerical control. Li-S cell SoC estimation can be categorized as a time series estimation problem.

This paper proposes a novel framework based on a LSTM RNN model for all Li-S state estimation; and within this framework a generic approach for SoC estimation for a new prototype Li-S battery cell is presented. The LSTM RNN is able to predict the SoC while maintaining accuracy and for 
varying initial conditions. By contrast with many published methods, this method does not require extensive battery cell modelling or good knowledge of initial SoC values. An appropriate level of balance between efficiency and functions has been achieved.

The paper will describe the full method including the acquisition of experimental data, the algorithm details, the training and testing methods, and the results. It will be shown that the estimator performs effectively on data obtained from realworld cells, despite an unknown initial condition.

\section{LI-S CELL CHARACTERIZATION AND ESTIMATION TASKS}

\section{A. Li-S cell and experimental test setup}

The lithium-sulfur cells used in this research are provided by OXIS Energy [2]; the company holds several key patents in Li-S battery manufacturing and related research. The battery research group at Cranfield University is in collaboration with OXIS Energy in multiple parallel projects from EU and UK, covering the areas of control, simulation, application, duty cycle modelling, hardware prototyping, mixed hardware/simulation test environments and state estimation.

In this paper the Li-S cells are tested for generating the experimental datasets for the training of LSTM RNN. The power source/sink devices located at the battery test laboratory of Cranfield University are used for carrying out the Li-S cell tests, where a duty cycle test current profile is the input signal and the terminal voltage is the output signal. The Li-S cell is put in an aluminum container which is placed in a temperature controllable thermal chamber, as shown in Figure 1.

\section{B. Training features of the Li-S cell}

In this paper, the measurements and observable variables serve as the training inputs for the LSTM RNN. The measurements include the current profile $I$ and terminal voltage measurement $V_{t}$. This Li-S cells have partly demonstrated normal performance as commercial Li-ion cells and partly demonstrated peculiar state properties according to Cranfield testsespecially a relatively flat voltage which is also known as 'low plateau', as shown in the red dash box (verses 'high plateau' in green dash box) in Figure 2. It has been proved to reduce the state observation level in $[10,11]$. The observable internal resistance $R$ is calculated based on the assumption of a resistance between two terminals of a single cell; the internal resistance is obtained from the voltage difference over the average current within a time window. The time window length is set at 75 seconds in this experiment if there is no extra clarification. The SoC value calculated through coulomb counting is used as the one training input. The LSTM RNN is constructed with three or four inputs in the experiments of this paper.

The tasks are (1) to predict the SoC value without parameterization of battery cell and without knowledge of initial SoC value, (2) to train a robust SoC estimation LSTM RNN considering the nonlinearities and new voltage-time property from the training data, and (3) to test the estimation performance of the LSTM RNN under multi-dimensional training features.

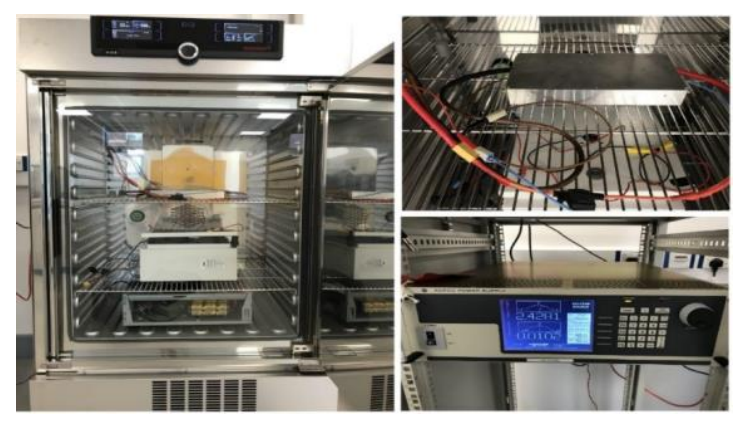

Figure 1. Experimental facilities
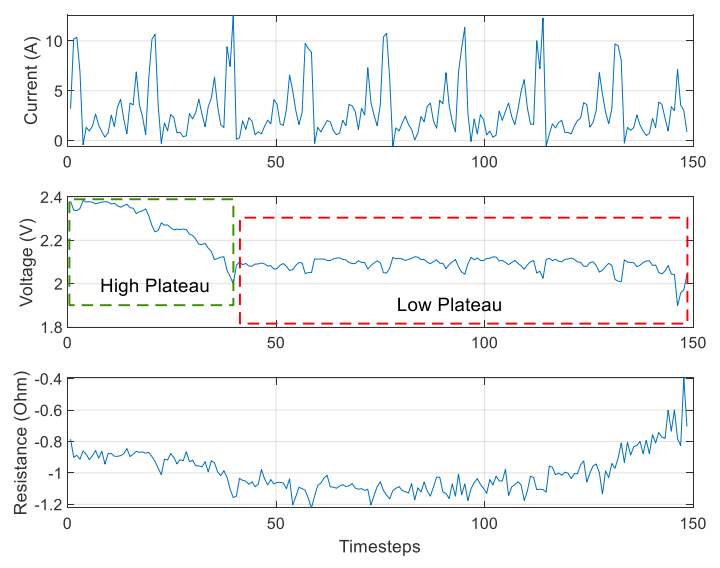

Figure 2. The training features

\section{TECHNICAL SOLUTION}

A. A data-based framework for all-state estimation

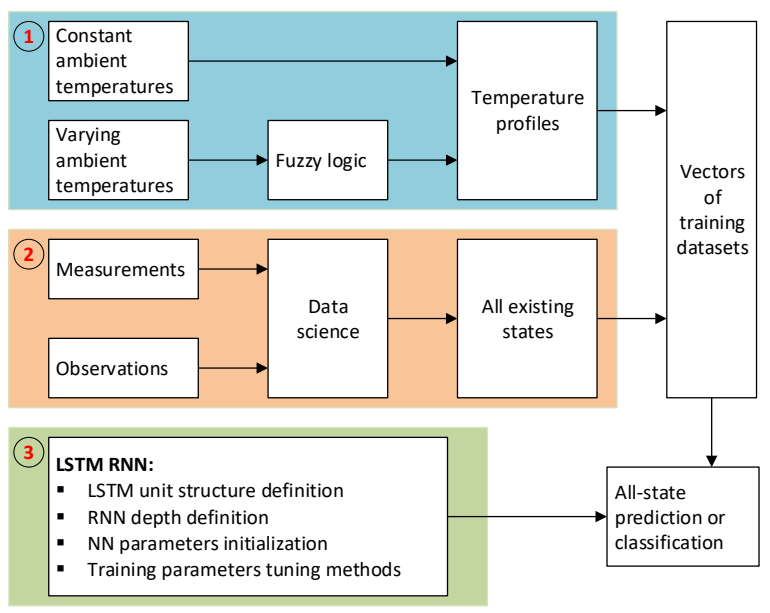

Figure 3. All-state estimation framework

Based on a broad review of related knowledge and research, we propose a framework for all-state estimation of Li$\mathrm{S}$ cell using LSTM RNN. As described in Figure 3, this model is capable of carrying out single-task estimation as well as 
multi-task estimation. In this paper, it is calibrated for SoC estimation of Li-S cell considering constant ambient temperatures, measurements, and LSTM RNN structure.

Part 1: LSTM RNN based on constant ambient temperature and varying ambient temperature

Since temperature acts as a key factor in $\mathrm{Li}-\mathrm{S}$ cell, i.e. lifespan, discharging rate, charging speed, and safety, it deserves to be researched as an independent part, as shown in Part 1 of Figure 3. Within this framework the temperature profile is composed of two parts: constant ambient temperatures and varying ambient temperatures. Firstly, the commercial thermal chambers and the emerging high-standard thermal management facilities have provided complementary capability in obtaining enriched temperature profiles at laboratory environment. A duty cycle test profile under constant temperature can be obtained easily, by repeating the test under different temperature levels it is easy to get an adequate amount of training data. Secondly, the sophisticated work conditions of vehicles have supplied with various temperature profiles by carrying out test under varying and difficult ambient temperatures, more useful nonlinear and uncertain scenarios within the measurements can be captured as well. The above two points have proposed both chance (more accessible datasets) and challenges (how to use the datasets?). For the varying temperature, fuzzy logic has provided an alternative probability method to determine a temperature scope within which a work scenario of Li-S cell may happen, though it needs to be considered together with electrical measurements and observations $[4,10]$.

\section{Part 2: LSTM RNN based on measurements and observa- tions}

The Part 2 of Figure 3 showed access to measurable and observable Li-S states (temperature excluded). A comprehensive use of measurements and observations have greatly enriched the dataset storage with more training features. For the purpose of building a full estimation model for Li-S performance and Li-S aging, the all-state estimation framework may consider training LSTM RNN using full features in the first instance, then consider distributed modelling for enhancement of local functions. Both routes work together to exploit the capability of LSTM RNN fully. In all the features, the linear features increase estimation accuracy and reduce error rate. The nonlinear features may act with double-edge effects, i.e. increase the complexity of decision making while enrich training features.

The electrical training features are obtained at constant ambient temperature in most laboratory study, while it is obtained at varying temperature at real-world test. According to different temperature profile or different work scenarios, the category for the same training features will be different. Therefore, the Part 2 in Figure 3 does exist out of Part 1.

Data science technologies, e.g. data mining and statistics, are be used in processing raw datasets together with physical or engineering theories, to relax requirements on ANN training.

Part 3: LSTM RNN based on optimal initialization of parameters and network structures

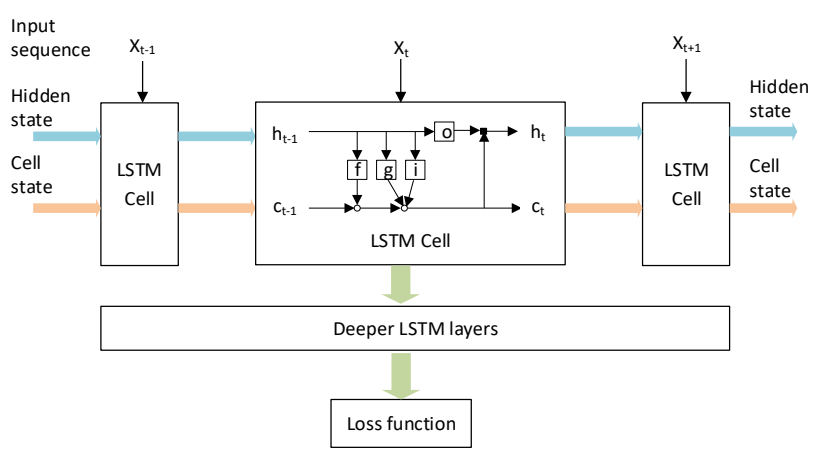

Figure 4. LSTM RNN structure

The Part 3 has been shown in the green area of Figure 3. This model works by using gradient-based techniques [12]. More specifically, this model is composed of the LSTM unit structure definition, RNN depth definition, ANN parameters initialization and training parameters tuning methods. In this research MATLAB Deep Learning Toolbox was used, which limited access to the LSTM unit structure definition. However, the remaining three parts can be realized conveniently. The ANN parameters initialization and the training parameters tuning methods are used in the task of Li-S cell SoC estimation.

This all-state prediction framework is designed at the critical development period of the battery industry; a systematic research around the estimation of long-term and temporary states of vehicle batteries at complex ambient and internal thermal conditions is desperately needed. The authors of this article is committed to structural design and system development in battery state and thermal management to help battery research, development and manufacturing with reduced resource consumption.

\section{B. LSTM RNN structure}

In this section, the LSTM RNN structure is introduced. The LSTM RNN has been proposed as a candidate for battery state estimation because of its capability in processing multi-dimensional data without equivalent circuit model (ECM) and the capability in learning long-term dependencies between time steps of sequence data $[4,12,13]$. The LSTM cells are embedded in the RNN framework with multiple layers, as shown in Figure 4. A LSTM RNN has overcome gradient decent from ANN application, which caused degradation of deep-learning performance. It is further proved as a part of the training algorithm in Section $C$.

In the LSTM RNN structure, the LSTM Cell calculates the hidden state (output state) and the updated cell state using the last time step of hidden state and the current time step of input sequence. A LSTM layer is used to process a sequence of data, in which the cell state functions as a storage pool of learned information from previous time steps of data while the hidden state represents the current output. In order to obtain high performance multi-layer LSTM cells are often used in training. A loss function is usually incorporated in the calculation process. The hidden state and cell state is selected, forgotten, and updated through the use of gate mechanism, which includes input gate, forget gate, and output gate. 


$$
\begin{aligned}
& i_{t}=\sigma_{g}\left(W_{i}\left[h_{t-1}, X_{t}\right]+b_{i}\right)=\sigma_{g} \operatorname{net}_{(i, \sim)} \\
& f_{t}=\sigma_{g}\left(W_{f}\left[h_{t-1}, X_{t}\right]+b_{f}\right)=\sigma_{g} \operatorname{net}_{(f, \sim)} \\
& g_{t}=\sigma_{c}\left(W_{g}\left[h_{t-1}, X_{t}\right]+b_{g}\right)=\sigma_{g} n e t_{(g, \sim)} \\
& o_{t}=\sigma_{g}\left(W_{o}\left[h_{t-1}, X_{t}\right]+b_{o}\right)=\sigma_{g} n e t_{(o, \sim)} \\
& c_{t}=f_{t} \odot c_{t-1}+i_{t} \odot g_{t} \\
& h_{t}=o_{t} \odot \sigma_{c}\left(c_{t}\right)
\end{aligned}
$$

where the $W$ denotes weight matrices, $X$ is the input sequence, $i, f, o, h, c, b$ represents the input gate, forget gate, output gate, hidden state, cell state, and the bias respectively, $\odot$ is the element-wise product of the vectors. The net $t_{(*, \sim)}$ are weight inputs. The sigmoid function $\sigma(x)$ is given by $\sigma(x)=\frac{1}{1+e^{-x}}$.

\section{LSTM RNN training algorithm}

The LSTM has been proved to be effective in estimation tasks for long-time sequence datasets, since it introduced a new weight gradients processing mechanism which distinguish it from other ANN methods [13, 14]. The following algorithm demonstrates the process by which gradient descent is eliminated. As a training algorithm, the LSTM includes both forward and backward calculation. The algorithm is stated as follows:

Step 1. Forward output calculation for neurons;

Step 2. Backward error calculation for neurons, which include back calculation along time and error transformation between layers;

Step 3. Calculate weight gradients from errors.

The forward calculation is shown in Equation (1), and the backward calculation of errors and weight gradients is shown in the following context. The error at time $t$ is defined as a derivative function of loss against output:

$$
\delta_{t}=\frac{\partial \operatorname{Loss}}{\partial h_{t}}
$$

The error corresponding to each gate are described as follows:

$$
\begin{aligned}
& \delta_{(i, t)}=\frac{\partial \operatorname{Loss}}{\partial \operatorname{net}_{(i, t)}} \\
& \delta_{(f, t)}=\frac{\partial \operatorname{Loss}}{\partial \operatorname{net}_{(f, t)}} \\
& \delta_{(g, t)}=\frac{\partial \operatorname{Loss}}{\partial \operatorname{net}(g, t)} \\
& \delta_{(o, t)}=\frac{\partial \operatorname{Loss}}{\partial \operatorname{net}_{(o, t)}}
\end{aligned}
$$

For the backward calculation, the error at time $t-1$ can be calculated from time $t$ :

$$
\delta_{t-1}=\delta_{t}^{T} \frac{\partial h_{t}}{\partial h_{t-1}}
$$

where $\frac{\partial h_{t}}{\partial h_{t-1}}$ is a Jacobian Matrix. The backward error with long time-step is obtained by putting Equation (1) into (4):

$$
\delta_{t-1}=\sum_{\epsilon:=\{i, f, g, o\}} \delta_{(\epsilon, t)}^{T} \frac{\partial n e t_{(\epsilon, t)}}{\partial h_{t-1}}
$$

Based on Equation (5), the forward error is calculated:

$$
\delta_{k}=\prod_{\tau=k}^{t-1} \delta_{\tau}
$$

Assume that the above calculation is for the lay- $n$ in LSTM RNN, we define the error to an upper LSTM layer:

$$
\delta_{t}^{n-1}=\frac{\partial \text { Loss }}{n e t_{t}^{n-1}}
$$

The weight gradients at each time step are computed using the errors at each time step. Different from traditional approach, the accumulation is obtained as the final weight gradient:

$$
\begin{aligned}
& \frac{\partial \text { Loss }}{\partial W_{(i, t)}}=\sum_{j=1}^{t} \frac{\partial \operatorname{Loss}_{(i, j)}}{\partial \operatorname{net}_{(i, j)}} \\
& =\sum_{j=1}^{t} \delta_{(i, j)} h_{j-1}^{T} \\
& \frac{\partial \text { Loss }}{\partial W_{(f, t)}}=\sum_{j=1}^{t} \frac{\partial \operatorname{Loss}_{(f, j)}}{\partial \operatorname{net}_{(f, j)}} \\
& =\sum_{j=1}^{t} \delta_{(f, j)} h_{j-1}^{T} \\
& \frac{\partial \text { Loss }_{(g, t)}}{\partial W_{(j=1}}=\sum_{j}^{t} \frac{\partial \operatorname{Loss}_{(g, j)}}{\partial n e W_{(g, j)}} \\
& =\sum_{j=1}^{t} \delta_{(g, j)} h_{j-1}^{T} \\
& \frac{\partial \text { Loss }}{\partial W_{(o, t)}}=\sum_{j=1}^{t} \frac{\partial \text { Loss }_{(o, j)}}{\partial \operatorname{net}_{(o, j)}} \frac{\partial \operatorname{net}_{(o, j)}}{\partial W_{(o, j)}} \\
& =\sum_{j=1}^{t} \delta_{(o, j)} h_{j-1}^{T}
\end{aligned}
$$

Correspondingly, the bias gradients are obtained the same way as weight gradients:

$$
\begin{aligned}
\frac{\partial \text { Loss }}{\partial b_{(i, t)}} & =\sum_{j=1}^{t} \delta_{(i, j)} \\
\frac{\partial \text { Loss }}{\partial b_{(f, t)}} & =\sum_{j=1}^{t} \delta_{(f, j)} \\
\frac{\partial \text { Loss }}{\partial b_{(g, t)}} & =\sum_{j=1}^{t} \delta_{(g, j)} \\
\frac{\partial \operatorname{Loss}}{\partial b_{(o, t)}} & =\sum_{j=1}^{t} \delta_{(o, j)}
\end{aligned}
$$


Finally, gradients corresponding to input layers $X_{t}$ are calculated:

$$
\begin{aligned}
& \frac{\partial \text { Loss }}{\partial W_{\left(i, X_{t}\right)}}=\delta_{(i, t)} X_{t}^{T} \\
& \frac{\partial \operatorname{Loss}}{\partial W_{\left(f, X_{t}\right)}}=\delta_{(f, t)} X_{t}^{T} \\
& \frac{\partial \operatorname{Loss}}{\partial W_{\left(g, X_{t}\right)}}=\delta_{(g, t)} X_{t}^{T} \\
& \frac{\partial \operatorname{Loss}}{\partial W_{\left(o, X_{t}\right)}}=\delta_{(o, t)} X_{t}^{T}
\end{aligned}
$$

\section{Evaluation metric for SoC estimation accuracy}

The estimation accuracy of the time series is used as an evaluation metric for the estimation accuracy of the battery SoC. There are several types of evaluation metrics to prove the performance from various angles, however in the application scenario of this paper the estimation accuracy is the only evaluation metric [15]. The Root Mean Square Error (RMSE) is chosen to evaluate the performance. A lower RMSE value indicates a better estimation performance. It is defined in the equation (11).

$$
R M S E=\sqrt{\sum_{i=1}^{n}\left(A_{i}-P_{i}\right)^{2} / n}
$$

where $A_{i}$ is the real value, $P_{i}$ is the estimation, $n$ is the number of samples.

\section{EXPERIMENTS}

\section{A. Training}

The training is realized by designing a multi-layer LSTM $\mathrm{RNN}$ for a regression process. In order to avoid the gradient vanishing, the Adam optimization is used.

The overall performance of Li-S SoC estimation is a complex tuning process of all the relevant parameters of LSTM RNN, where beneficial effects of one characteristic are often established at the expense of other characteristics. In order to highlight the influence of fixed parameters on LSTM RNN,
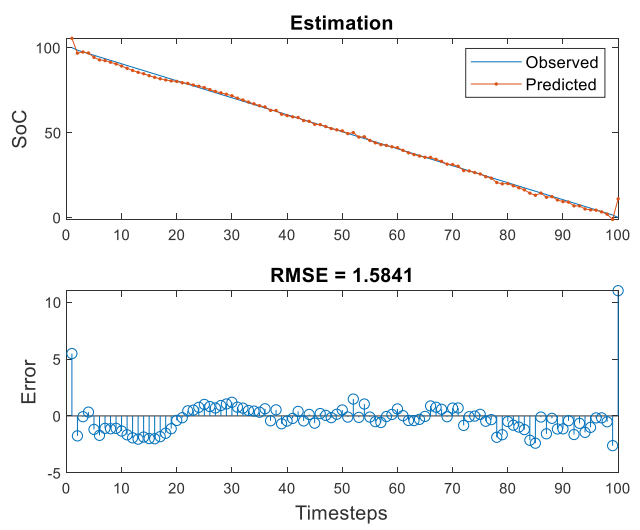

Figure 5. SoC estimation of Type-1 Li-S cell one variable value is tuned at one time when other parameters are unchanged to form comparable experiments. For the convenience of balancing between calculation speed and accuracy, the duty cycle test data are incorporated into training inputs using non-overlapping time windows. The time window refers to the time intervals within which a number of sampled values are averaged and appropriately smoothed. The sampling time of Cranfield battery test facilities is $1 \mathrm{~s}$ and the minimum change of battery SoC is set at $1 \%$ in the algorithm, which secured enough time for various lengths of time windows in experiments. In this research the $25 \mathrm{~s}, 50 \mathrm{~s}, 75 \mathrm{~s}$, and $100 \mathrm{~s}$ have been used in the study though only $75 \mathrm{~s}$ is chosen in accordance with acceptable efficiency and accuracy.

\section{B. Basic regression-based SoC estimation for two types of battery}

The measured datasets have been obtained from WLTP tests performed at constant $20^{\circ} \mathrm{C}$ temperature and $100 \%$ SOC levels. Two types of batteries are tested both at appropriate stage of lifetime, generally within 20 cycles of charge/discharge. Type-1 cell is manufactured with capacity of $12 \mathrm{Ah}$ and Type-2 14Ah. In order to achieve higher energy capacity, the manufacturing composition of the Type- $2 \mathrm{Li}-\mathrm{S}$ cell is different from that of the Type-1, and thus the internal resistance characteristic of the second battery shows strong nonlinearity and irregularity [4]. The internal resistance property is hugely different between these two types of cells.

From Figure 5 and Figure 6, Type-1 and Type-2 Li-S cells are tested under the same condition-three training features $I, V_{t}$ and $R$, a 75-second time window and the initial learning rate 0.02 . The experimental results show that the SoC estimation accuracy for Type- 2 cell is much lower than that in Type1 , which is caused by the loss of linearity of the internal resistance in Type-2 Li-S cell. However, the result has presented a certain degree of robustness against nonlinearity since the SoC estimation results are given out with acceptable level of estimation error for both types of Li-S cell.

In order to make this case a generic study on SoC estimation based on the LSTM RNN, the Type-2 Li-S cell is chosen as the experimental objective under different circumstance in the following context.
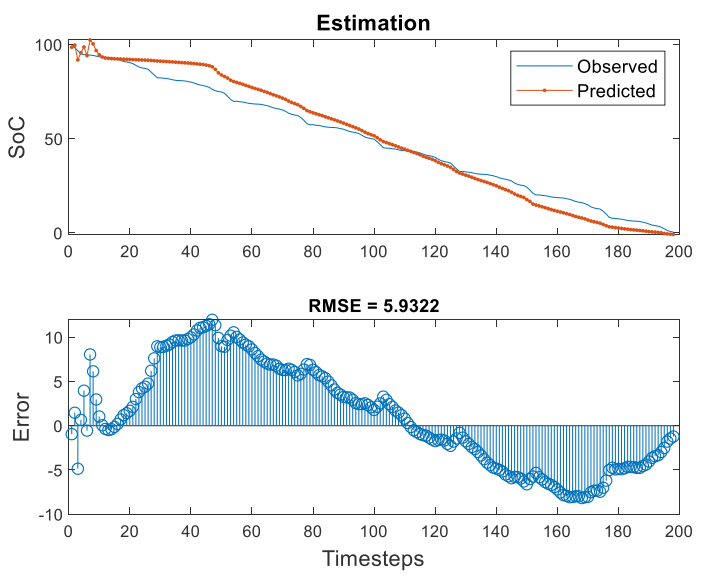

Figure 6. SoC estimation of Type-2 Li-S cell 


\section{SoC estimation with two features and three features}

A dataset with multiple training features is essential since they bring in complementary information for a prediction task. Ideally these features show smooth and low-noise with clear developing trends. The 'high plateau' in Figure 2 supplied a positive example, while the 'low plateau' voltage supplied with almost void information and the resistance noisy and highly-irregular information. Therefore, a multiple-feature test is applied to find out the effectiveness from features $I, V_{t}$ and additional feature $R$, in order to verify (1) $I, V_{t}$ are effective in prediction and (2) $R$ is ineffective as a nonlinear and highly noisy additional feature in prediction task.

The measured datasets from this experiment have been obtained in response to an extensive range of charge/discharge WLTP tests performed at constant $15^{\circ} \mathrm{C}$ temperature and $100 \%$ SOC levels. The datasets measured from Type-2 Li-S cell WLTP tests are used for the LSTM RNN training using the proposed method - one with two features $\left(I, V_{t}\right)$ and the other with three features $\left(I, V_{t}\right.$ and $\left.R\right)$. They have been tested under the same condition - $\mathrm{a} 75 \mathrm{~s}$ time window and an initial learning rate 0.02 .

In this experiment, the results from the two-feature-training as shown in Figure 7 have even shown a higher accuracy than that of the three-feature-training as shown in Figure 8. It is verified that the newly introduced a third training featurethe internal resistance with strong nonlinearity from the Type$2 \mathrm{Li}-\mathrm{S}$ cell does not help with improving training/test performance of the LSTM RNN. Without the third feature, the twofeature-training is proven to have maintained a good estimation performance, reduced the training time (Empirically, over
$50 \%$ ) and improved the calculation efficiency. This section has set up a standard structure with fixed dimensions for SoC estimation using LSTM RNN.

\section{SoC estimation with relaxed initial $\mathrm{SoC}$}

The measured datasets from this experiment have been obtained in response to an extensive range of charge/discharge WLTP tests performed at constant $20^{\circ} \mathrm{C}$ temperature and various SOC levels. The data measured from Type- $2 \mathrm{Li}-\mathrm{S}$ cell WLTP test are used for LSTM RNN training using two features $\left(I, V_{t}\right)$. The experiment is conducted with a 75 -second time window and an initial learning rate 0.05 .

In this experiment, the training performance of the algorithm with relaxed initial SoC are demonstrated. In other words, the initial $\mathrm{SoC}$ values are not required in the training and test. Therefore, a random initial value $(100 \%, 80 \%, 60 \%$, $30 \%$ ) is set in this experiment in order to examine the SoC estimation performance under random initial SoC. This results showed a highest RMSE value of $28.5861 \%$ when the initial $\mathrm{SoC}$ is set at $30 \%$. The experiment shows the remarkable capability of LSTM RNN in estimation of Li-S cell SoC without considering initial SoC.

The results have showed that there is a SoC dip when SoC dropped into the scope between $75 \%$ and $70 \%$, and it recovers quickly to an acceptable estimation value. This phenomenon can be found out in the Figure 9 and 10. The SoC dip is caused by the voltage dip at the transition between the high discharge plateaus and the lower discharge plateaus, who contribute about $25 \%$ and $75 \%$ to the total discharge capacity respectively.

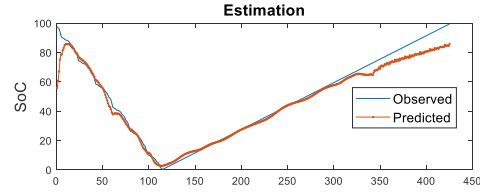

RMSE $=\mathbf{5 . 6 6}$

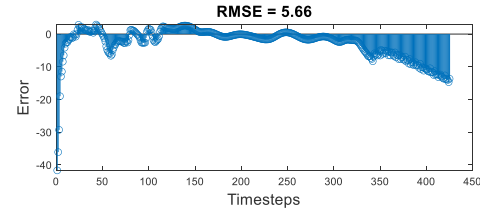

Figure 7. SoC estimation with two features

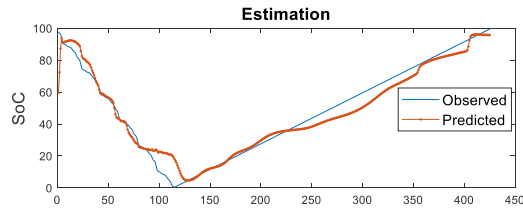

RMSE $=\mathbf{5 . 9 8 6 5}$

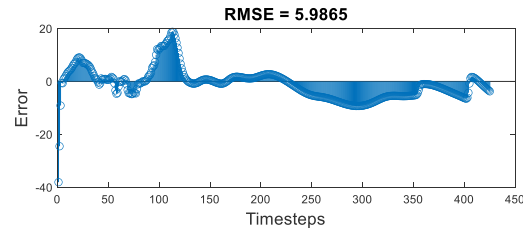

Figure 8. SoC estimation with three features
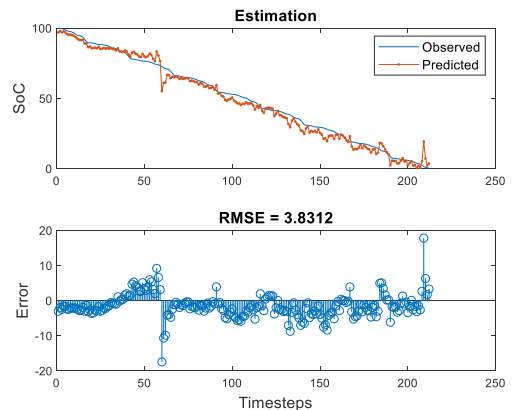

Figure 9. Initial SoC 100\%
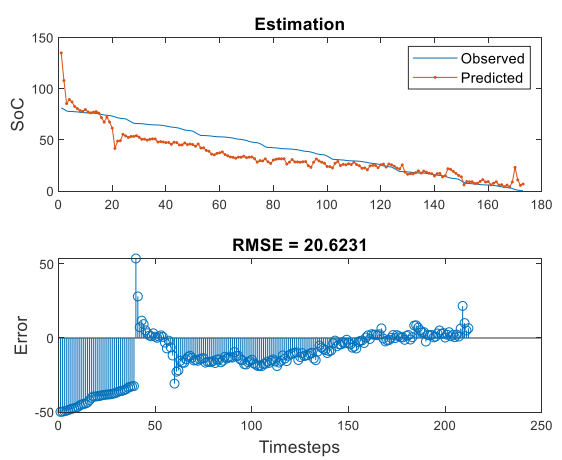

Figure 10. Initial SoC $80 \%$ 

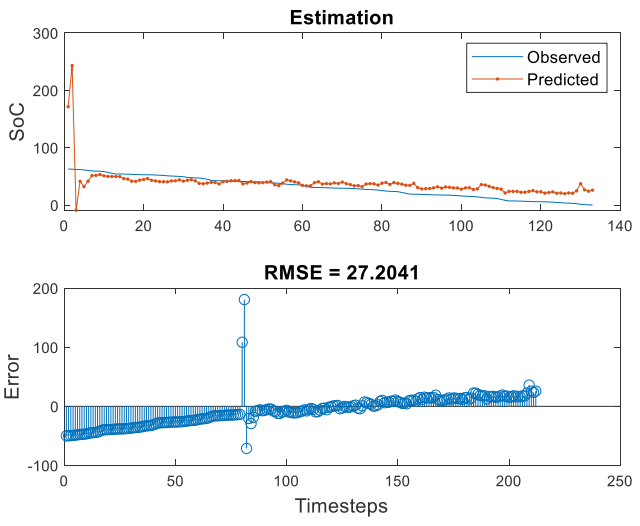

Figure 11. Initial SoC $60 \%$
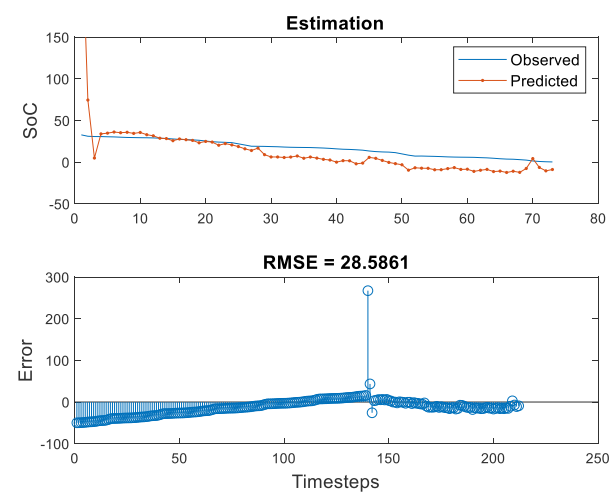

Figure 12. Initial SoC $30 \%$

The SoC estimation curves in the Figure 11 and 12 showed a fast convergence process at start. It is due to the relaxed initial SoC in both training and test, the SoC estimation depends on the long-term memory between time steps of sequence data. A convergence process in the start is unavoidable. This distinguish LSTM RNN from other ANNs with capability in processing long time sequence data.

\section{CONCLUSION}

In this paper, the following contributions have been presented for the state estimation of Li-S cells using LSTM RNN in the context of WLTP test.

1) An all-state estimation framework using LSTM RNN model for Li-S battery management has been proposed. It has integrated the measurements and observations from field tests for Li-S state estimation.

2) The LSTM RNN model has been calibrated to estimate $\mathrm{SoC}$ for fresh prototype Li-S cells. This approach has removed dependency on ECN model approaches and brought in estimation capability through learning longterm dependencies between time steps of sequence data. The estimation model has given reliable estimation performance despite the challenges presented by the near-flat 'low plateau' open-circuit voltage.
3) A LSTM RNN structure adopting the Adam solver was described for the purposes of training and testing with the experimental datasets. The results demonstrated that this method is robust to uncertainties and nonlinearities to a certain degree.

4) This method has showed satisfactory SoC estimation performance for fresh prototype Li-S cells with new electrochemical properties, and there is balanced estimation accuracy and convergence speed through SoC estimation. Some critical constraints have been relaxed, e.g. the system parameterization and initial $\mathrm{SoC}$ values.

The authors are working towards multi-task estimation and all-state estimation for Li-S cell management using deep LSTM RNN.

\section{REFERENCES}

[1] UK Research and Innovation, The Faraday Battery Challenge: funded projects to date. UK, Sep. 2019.

[2] OXIS Energy, 'Our Cell and Battery Technology Advantages', https://oxisenergy.com/technology/. Accessed Sep. 2019.

[3] A. Fotouhi, D. Auger, L. O'Neill, T. Cleaver and S. Walus, "LithiumSulfur Battery Technology Readiness and Applications-A Review," Energies, 10 (12), 2017.

[4] M. Wild, G. Offer, Lithium-Sulfur Batteries. UK: Wiley-Blackwell, 2019.

[5] R. R. Richardson, S. Zhao, and D. A. Howey, "On-board monitoring of 2-D spatially-resolved temperatures in cylindrical lithium-ion batteries: Part II. State estimation via impedance-based temperature sensing," Journal of Power Sources, vol. 327, pp. 726-735, 2016.

[6] M. Chen, G. A. Rincon-Mora, "Accurate electrical battery model capable of predicting runtime and I-V performance," IEEE Transactions on Energy Conversion, 21 (2), pp. 504-511, 2006.

[7] D. P. Finegan, M. Scheel, J. B. Robinson, B. Tjaden, I. Hunt, T. J. Mason, J. Millichamp, M. Michiel, G. J. Offer, G. Hinds, D. J.L. Brett and P. R. Shearing, "In-operando high-speed tomography of lithiumion batteries during thermal runaway," Nature Communications, vol. 6, 2015.

[8] T. Bruen, J. Marco, "Modelling and experimental evaluation of parallel connected lithium ion cells for an electric vehicle battery system," in Journal of Power Sources, vol. 310, pp. 91-101, 2016.

[9] T. Zhang, M. Marinescu, L. O’Neill, M. Wild, and G. Offer, "Modeling the voltage loss mechanisms in lithium-sulfur cells: the importance of electrolyte resistance and precipitation kinetics," Royal Society of Chemistry, vol. 17, pp. 22581-22586, 2015.

[10] A. Fotouhi, D. J. Auger, K. Propp, and S. Longo, "Lithium-Sulfur Battery State-of-Charge Observability Analysis and Estimation," IEEE Transactions on Power Electronics, 33 (7), pp. 5847-5859, 2018.

[11] A. Fotouhi, D. J. Auger, K. Propp, and S. Longo, "Electric vehicle battery parameter identification and SOC observability analysis: $\mathrm{NiMH}$ and Li-S case studies," IET Power Electronics, 10 (11), pp. $1289-1297,2017$.

[12] Y. Oussar, G. Dreyfus, "Initialization by selection for wavelet network training," Neurocomputing, 34 (1-4), pp. 131-143, 2000.

[13] M. Andrychowicz, M. Denil, S. G. Colmenarejo, M. W. Hoffman, D. Pfau, T. Schaul, B. Shillingford, and N. Freitas, "Learning to learn by gradient descent by gradient descent," in Proc. Conference on Neural Information Processing Systems, Barcelona, Spain, Dec. 2016.

[14] H. Harutyunyan, H. Khachatrian, D. C. Kale, G. Ver Steeg, and A. Galstyan, "Multitask learning and benchmarking with clinical time series data," Scientific Data, vol. 96, 2019.

[15] W. He, N. Williard, C. Chen, and M. Pecht, "State of charge estimation for $\mathrm{Li}$-ion batteries using neural network modelling and unscented Kalman filter-based error cancellation," Electrical Power and Energy Systems, vol. 62, pp. 783-791, 2014. 